\title{
Treatment of Ankylosis in Children Associated With Osteogenic Distraction: Literature Review and Case Report
}

João Luiz Carlini, DDS, PhD, $\mathrm{Ms}^{1 *}$, Ian Luna Parente Brasileiro, $\mathrm{DDS}^{2}$, Andrea Duarte Doetzer, $\mathrm{DDS}^{2}$, Michelle Fernanda Fast, DDS ${ }^{2}$

\author{
${ }^{1}$ Associate Professor UFPR Cirurgia I \\ ${ }^{2}$ Oral and Maxillofacial Surgeon, Hospital Evangelico of Curitiba, Alameda Augusto Stellfeld, 1908 - Bigorrilho, Curitiba - PR, 80730-150, Brazil
}

DOI: $10.36348 /$ SJODR.2019.v04i10.007 $\quad$ | Received: 11.10.2019| Accepted: 18.10.2019| Published: 27.10 .2019

*Corresponding author: João Luiz Carlini, DDS, $\mathrm{PhD}, \mathrm{Ms}$

\section{Abstract}

Introduction: Temporomandibular joint ankylosis is defined as a bone or fibrous adhesion of the anatomical joint and its components in the glenoid fossa, and it is accompanied by limited mouth opening, causing difficulties in chewing, speaking and oral hygiene, which may influence mandibular growth. This condition in children may or may not be associated with a disease, syndrome or trauma. Method: To review the literature on the treatment of ATM ankylosis associated with micrognathia in children with osteogenic distraction, and to report two clinical cases of children with unilateral or bilateral ankylosis with mandibular micrognathia. Methods: To present a broad research on the etiology, risk factors and complications of this condition as the options of treatments for the ankylosis of TMJ in children by Buccomaxillofacial Surgery treated with gap arthroplasty surgery without interposition of materials, with the installation of osteogenic distractors for the correction of mandibular deformities. Results: The cases presented here continue to be treated and monitored since their development is not yet complete, but the procedures performed are successful without presenting recurrences so far. Conclusions: Osteogenic distraction is one of the treatments that can treat ankylosis in children with success. However, the successful treatment of children with TMJ ankylosis with dentofacial deformities is slow and prolonged, and the earlier it is initiated, the greater the success of treatment completion, always depending on the joint work of a multidisciplinary team, as well as family support and patient collaboration.

Keywords: ankylosis, osteogenic distraction, TMJ.

Copyright @ 2019: This is an open-access article distributed under the terms of the Creative Commons Attribution license which permits unrestricted use, distribution, and reproduction in any medium for non-commercial use (NonCommercial, or CC-BY-NC) provided the original author and source are credited.

\section{INTRODUCTION}

Ankylosis of the temporomandibular joint is defined as bone or fibrous adhesion of the anatomical joint and its components in the glenoid fossa, and is accompanied by limited opening of the mouth, causing difficulties in chewing, speech and oral hygiene, and may influence mandibular growth. This condition in children may or may not be associated with disease, syndromes or trauma. Its treatment is extremely challenging to oral and maxillofacial surgery, requiring the association of one or more surgical interventions, among them total or partial arthroplasty of the TMJ for its release, osteogenic distraction for the development of the mandible and, later, the replacement of the TMJ by total prosthesis and orthognathic surgery when necessary. The initiation of early surgical treatment, even in childhood, aims to improve mouth opening, feeding, diction and oral hygiene, as well as promote functional and aesthetic growth of the child, minimizing psychosocial problems associated with deformity during growth. In this paper we will discuss the etiologies of ATM ankyloses, treatment options and the use of bone distraction. Two clinical cases of children with TMJ ankylosis with mandibular micrognathia treated at CAIF (Integral Center of Cleft Patients, Curitiba / Pr/Brazil) will be described, with arthroplasty surgery with segment resection, with no interposing of materials concomitant and with the use of osteogenic distraction of the mandible branch. Therefore, the aim of this study was to review the literature on the treatment of ankylosis in children with a mandibular distractor.

\section{LITERATURE REVIEW Ankylosis of TMJ}

The temporomandibular joint (TMJ) is one of the most important joints in the human body. It consists of a condylar process of the mandible, temporal fossa (mandibular), articular disc and articular capsule. The term "ankylosis" is of Greek origin (from the Greek 
word agkuloV: bent or crooked) and corresponds to a "rigid joint" since it leads to a partial or total loss of TMJ mobility $[1,31]$. The surgical treatment of ATM ankylosis is a set of highly controversial subjects.

\section{Types of Ankylosis}

Sawnhey by Turlington and Durr [2] divided into 4 classes the ATM ankylosis according to the degree of joint destruction.

- Type I: The head of the condylar process is visible, but significantly deformed, with the presence of fibroadhesion.

- Type II: Consolidation of the deformed head of the condylar process and articular surface, mainly in the anterior and posterior borders of the structures, and the medial part of the condyle remains intact;

- Type III: Fused bone mass involves the mandibular branch and zygomatic arch, the atrophic fragment displaced from the anterior part of the condyle is located medially.

- Type IV: The TMJ is completely destroyed by the growth of bone mass between the mandibular branch and the cranial base.

Taking into account heterotopic bone formation within the ankylotic mass, Turlington and Durr $^{2}$ classified ATM ankylosis into:

- Grade 0: No visible bone island;

- Grade 1: Bone islands visible in soft tissue around the joint;

- Grade 2: periarticular bone formation;

- Grade 3: apparent ankylosis.

Grades 1, 2 and 3 are further classified as symptomatic and asymptomatic. Symptomatic ossification includes: Severe pain, decreased mouth opening (15 $\mathrm{mm}$ or less), total blockage or lateral decrease of the mandibular movements $[2-6,8]$.

\section{Etiology}

The most common causes of ATM ankylosis include trauma and local or systemic infections. The incidence of ankylosis due to infection has decreased considerably in recent years due to the evolution of antibiotic therapies. Perinatal trauma is considered the most common in children.

The underlying factors include: damage to the temporomandibular disc, age less than 10 years, and prolonged immobilization of the mandible after intracapsular trauma $[4,7,9,10]$.

Other etiologic factors include: ossific myositis, osteochondroma, rheumatoid arthritis, ankylosing spondylitis (Bechterew's disease), psoriatic arthritis, lupus erythematosus, radiotherapy, TMJ surgical treatment and possible complication after orthognathic surgery.
The pathogenesis of ectopic bone formation is unknown. Pluripotential mesenchymal cells are stimulated to differentiate into osteoblastic and chondroblastic cells in an unknown stimulation mechanism, with the bone matrix as the most likely agent [11-13].

ATM ankylosis may occur during development or after completion of growth. Thus, there are 4 groups of patients that can be affected distinctly [3]:

- Growing patients without dentofacial deformities;

- Growing patients with dentofacial deformities;

- Adults without dentofacial deformities;

- Adults with dentofacial deformities.

Each of these 4 groups requires individual treatment because of their specific character and different clinical manifestations.

\section{Clinical Aspects and Complications}

The infantile ankylosis developed during childhood is one of the most complex and can lead to deformity and impairment of mandibular growth, feeding difficulties, chewing and swallowing, difficulties in speech, and may result in dental caries and periodontal disease due to the difficulty of oral hygiene $[14,15]$. They even negatively affect the eruption and position of the teeth.

In relation to the face the patient is generally described as "bird profile", where the lower third of the face is considerably shortened and the deficient mandible is visibly retruded, the cervical angle is obtuse and the nasolabial angle is more open than normal $[3,4$, 16]. The face is asymmetrical with the chin significantly diverted to the affected side. It is also observed the lip incompetence and as the child grows, the face becomes increasingly asymmetrical due to limited mobility of the mandible, impairment of growth and function (malnutrition) and oral hygiene. Prolonged ankylosis leads to muscle atrophy, secondary elongation and hypertrophy of the coronoid process and consequently aesthetic impairment $[17,18]$.

In addition to causing physical changes in appearance, the condition has a serious negative effect on psychosocial development in young patients, who often develop depressive disorders because of the aesthetic appearance and functional difficulty they present.

\section{Treatment of ATM Ankylosis}

The first steps in the development of methods of surgical treatment of the TMJ affected by ankylosis were described in 1851. Between the 1850s and 1860s, they used the condilectomy and arthroplasty, generating space between the stumps (gap) interposing myofascial 
flaps, techniques still used in the resection of ankylosis $[2,19,20]$.

We currently have several techniques for the treatment of ankylosis and for the reconstruction of the joint. It can be performed at the same surgical time as resection of the ankylar mass or at another time [4].

The surgical treatment procedures currently used include:

Removal of Ankylosis:

- Condilectomy;

- Arthroplasty of the joint cavity;

- Gap arthroplasty with interposition of temporal myofascial flap with simultaneous unilateral coronoidectomy on the affected side or bilateral coronoidectomy;

All procedures are accompanied by physical therapy to improve mobility and stimulate all muscles.

In the Reconstruction Of The Articulation

- Arthroplasty with costochondral graft;

- Autogenous graft vascularized or not of sternum-clavicular joint, tibia or iliac crest.

- Reconstruction of the joint using an alloplastic prosthesis;

- Osteogenic distraction of the branch and body of the mandible on the affected side.

A necessary complement of surgical treatment is physical therapy (intensive exercise of opening the mouth). Based on the functional matrix theory of Moss [2, 22-24], surgical and functional restoration of both adjacent joint and adjacent soft tissues releases the growth potential of the mandible and may prevent the development of the deformity.

\section{DO in the Treatment of ATM Ankylosis Associated With Micrognathia - Surgical Techniques}

Osteogenic distraction is a widely used surgical technique in the treatment of ATM ankylosis associated with micrognathia for the correction of facial asymmetry. It may be applied alone or concomitantly to other ankylosis-related TMJ release and / or repair techniques, and may be used uni or bilaterally $[25,26]$.

Rao K et al., [18], described the use of the DO technique together with the gap arthroplasty at the same surgical time in children with ATM ankylosis associated with micrognathia. Eight children with a mean age of 8.5 years, with a mean mouth opening of $1.6 \mathrm{~mm}$ and facial asymmetry were treated. No material was presented in the gap arthroplasty and the osteotomy of the ankyrotic mass was approximately $2 \mathrm{~cm}$, in addition to the planing of the coronoid processes. The distractors were installed in the osteotomies performed in the region of the mandibular body, fixed with the long axis of the distractors parallel to the occlusal plane. The mean time of distraction was 21 days, beginning the physiotherapy of buccal opening in the first 24 postoperative hours. In all cases an over-correction of 5 mm was applied. At the end of the consolidation period, 6-8 weeks, the distractors were removed and the result in all cases was correction of the mandibular deformity and enlargement of the buccal opening to an average of $28 \mathrm{~mm}$ with active movement for chewing, swallowing and diction.

A variation of the technique was described in a study by Sadakah AA et al., [5] in 2006, in a group of 9 patients with a mean age of 19 years, with a mean mouth opening of $0.6 \mathrm{~mm}$, the performance of Le Fort I osteotomies and osteotomy of the mandibular branch with the installation of an osteogenic distractor for the correction of dento- facial before the release of ATM ankylosis, which was done in a second surgical time. The justification of the study was that a condition of the arthroplasty or gap arthroplasty prior to the correction of the deformity would cause instability in the movements caused by the DO and consequent correction of the impaired occlusion.

As the technique evolved, other technologies have been developed and associated with surgical procedures, increasing predictability and final stability, as well as facilitating planning [27, 28]. In 2010, Feiyun $\mathrm{P}$ et al., [8] described the use of three-dimensional craniofacial models in the planning of simultaneous surgical correction of atm ankylosis associated with mandibular micrognathia. Sixteen adult patients with mean age of 27 years were treated with bilateral ankylosis associated with micrognathia and obstructive sleep apnea or hypopnea. All patients underwent ATM arthroplasty with concomitant installation of mandibular branch and body distractors planned from simulation in 3D models, increasing the precision of corticotomies and positioning of the distractors.

Treatment approaches and sequences vary widely among authors, some include gap arthroplasty with or without material interposition, joint reconstruction with costochondral grafting, orthognatic surgery, and reconstruction with alloplastic prostheses [29, 30]. OD offers multiple benefits over other techniques, especially when combined with single-time arthroplasty [17, 31, 32]. Giraddi BG et al., [17] states that OD in children offers the option of stretching the mandible, providing room for dental eruption, occlusion correction and midline and facial asymmetry. The main objective of his study was to recommend the simultaneous interpositional arthroplasty to the DO, eliminating a second surgical act. The technique that used submitted 9 patients. The bone was removed by performing an osteotomy below the zygomatic arch and another about $1.5 \mathrm{~cm}$ below it and the block of bone mass was removed. A temporal myofascial flap was interposed in the gap, he said, filling the joint space prevents re-ankylosis. Through submandibular access, an oblique osteotomy was performed at the mandibular 
angle and the distractor was installed, so that its activation consented to vertical and lateral stretching of the mandible. Physical therapy was initiated 3-5 postoperative days and extended by 3 months. Distraction began on the 5th postoperative day until the correction of the midline with overcorrection of $2 \mathrm{~mm}$. After consolidation period of 12 weeks the distractor was removed. The total of the nine patients were followed clinically and radiographically for 3 years, presenting correction of facial asymmetry, correct eruption of the teeth and improved airway, giving the technique used greater benefits in relation to the other techniques described.

In an extra-oral approach, Bansal $\mathrm{V}$ et al., [31], published a study with 6 patients aged 4 to 8 years, all with unilateral post-traumatic ankylosis with micrognathia. The mean preoperative maximum interincisal opening was $3.5 \mathrm{~mm}$ without lateral and protrusive mandibular movements. The technique used was performed through an extra-oral incision at the posterior border of the lazy-S mandibular ramus exposing the ankylotic mass together with the ascending branch exposition. Gap arthroplasty was performed without any interposition of material at the site of resection. The coronoid processes were resected bilaterally and a reverse L-osteotomy was performed at the posterior border of the branch, approximately 25 $\mathrm{mm}$ below the notch and $10 \mathrm{~mm}$ anterior to the posterior border of the branch, defining this as the distraction transport disk. In this segment, the extra-oral distractors previously manufactured for this study were installed. After the latency period, the activation was started at $0.5 \mathrm{~mm}$ twice daily until it reached a contact of the transport disk with the glenoid fossa. The whole process was followed clinically and radiographically. After a 3-month consolidation period, CT scans showed a remodeling in the condylar region in all patients. The clinical and radiographic follow-up evaluation was performed for 13-27 months after the end of the activation period. With an average of 19 months of follow-up, the mean inter-incisal aperture was $29.1 \mathrm{~mm}$. Among the disadvantages observed in this technique are the scars on the face, the extended treatment duration and the discomfort for the patient with the position of the extra-oral device. However, advantageously, all patients tolerated the treatment well, presenting at the end of the study a desirable buccal opening, with no asymmetries on the face, with condylar remodeling and none presenting ankylosis recurrence.

\section{CASE REPORT}

Patient 1: VA, female, 7 years old, with no history of trauma, bilateral TMJ ankylosis with micrognathia, preoperative interincisal opening $=0 \mathrm{~mm}$. It was attended in the CAIF - Comprehensive Care Center to the Fissured Labiopalatal / PR in 2014 with a previous history of bilateral cost-chondral grafting performed in another service with recurrence of ankylosis. In 2015, she was submitted to surgery under general anesthesia, with nasotracheal intubation via fibrobroscoscopy, bilateral pre-auricular access was performed to remove total ankylotic mass of bilateral TMJ, without interposition of material. At the same time surgical was performed via bilateral Risdon access, bilateral osteotomy in mandibular branches with installation of bilateral intraoral bilateral osteogenic distractor. The intraoperative buccal opening reached $25 \mathrm{~mm}$. Physical therapy was started on the 3rd postoperative day and activation of the distractors started after 7 days. Approximately $4 \mathrm{~mm}$ overcorrection of the final activation was programmed. After 8 months after the consolidation period, it was submitted to the removal of distractors via bilateral Risdon access under general anesthesia. After 12 months of follow-up, the patient maintained a $15-\mathrm{mm}$ mouth opening and continued physical therapy. The patient is observed with annual consultations and no signs of relapse.

The patient continues the outpatient follow-up, maintaining a $21 \mathrm{~mm}$ opening. We are awaiting growth to assess the need for a new orthognathic surgery intervention.

Patient 2: VFTS, 4 years of age, presented at the CAF Buccomaxillofacial Service with complaint of mouth opening difficulty. At the clinical examination, we observed limitation of mouth opening $3 \mathrm{~mm}$, facial asymmetry. In previous history it was reported by parents that patient was born 8 months of gestation cesarean delivery because the mother presented preeclampsia. The patient remained in the ICU for 14 days due to respiratory problems. Those responsible did not know if there was a need for forceps at birth. They report that from the time she was born she had a "small" mouth and could not breastfeed. Even after discharge from hospital she was never able to nurse or eat properly because her mouth was "small". Based on this information, we can not state the origin of ankylosis, whether it is congenital or traumatic. In 2016 she was submitted to surgery under general anesthesia with nasotracheal intubation fibrobroncoscópica, through left pre-auricular access resection of ankylotic mass was performed without interposition of material. By access of Risdon was exposed the left mandibular branch where it was osteotomized and installed osteogenic distractor. Via intraoral access, contralateral coronoidectomy was performed. Activation was started after 7 days. After 12 weeks of consolidation, the patient was submitted to a new surgical procedure, via left Risdon access to reposition the distractor in order to continue the elongation of the left mandibular ramus. The patient was not collaborative with physiotherapy and had recurrence of ankylosis. A third surgical intervention was necessary after 10 months of the second, for new resection of the ankylosis, without interposition of material, via left pre-auricular access. The distractor was again repositioned in the mandibular ramus to continue its elongation, via Risdon access, and 
by iso-lateral coronoidectomy by intraoral access. In May 2018 the patient underwent removal of the distractor under general anesthesia and continued with physical therapy.

\section{DISCUSSION}

Correction of complex craniofacial deformities in antero-posterior, transverse and vertical planes is one of the most challenging problems in oral and maxillofacial surgery [4-7]. Among the causes of deformities are congenital craniofacial scoliosis, hemifacial microsurgery, Nager syndromes, Pfeiffer and Pierre Robin, all with multifocal involvement such as mandibular hypoplasia, facial asymmetry with deformation of the medial and midline to the affected side, hypoplasia of soft tissues associated with micrognathia. Such deformities may also result from external factors such as mandibular growth deficits resulting from condylar fractures and temporomandibular joint anchylosis or local infections suffered at an early age, causing considerable aesthetic deformity and functional disability. All these defects require treatments with interventions to achieve functional growth as well as the quality of final aesthetics [9-11, 33-35].

The classification of ATM ankylosis is a reasonable guide to choosing a treatment method. According to Yang's classification ${ }^{16}$, based on the reconstruction of coronal computed tomography, there are two types of ankylosis: with or without residual condyles. In ankylosis with residual condyles presenting laterally the bone fusion, the residual condyle should be maintained during surgery and only the lateral bone mass should be removed (TMJ lateral arthroplasty). This is especially important for growing children because the condyles preserved, these can develop and remodel later. For ankylosis without residual condyles, joint reconstruction by autogenous bone grafts, alloplastic TMJ prostheses or osteogenic distraction should be employed after the release of the bone fusion, and may be performed at the same time in surgery or in a second time [36-39].

Skeletal deformities caused by ankylosis were managed in the past with osteotomies in the mandible or maxilla or both, followed by acute orthopedic movements and skeletal fixation, with or without interposition of bone grafts. However, these treatment modalities have several limitations, such as the impaired vitality of erupting or erupting teeth, neurological incompetence, and the inability of the muscles and soft tissues to be acutely adapted, with consequent relapse [24, 25, 40]. In addition, donor site morbidity in autogenous bone grafts, or the high rejection rate of xenografts and prosthetic implants, which only aesthetically camouflage the defects without restoring the correct function were considerable. Such techniques did not allow full bone sculpting, that is, changing the bones shape to correct three-dimensional defects [23, 25, 26, 32, 33].
In recent years, alloplastic TMJ prostheses have been widely used in Europe and the United States. These are considered stable because they do not suffer resorption in comparison with autogenous bone grafts. There are standardized prostheses, but they are little used since the customized prostheses are available and are much easier to be implanted intraoperatively. In order to make the bone suitable for prostheses accurately, a computer-aided technique is used to guide the bone trimming and prosthesis implantation $[16,19$, 21].

$\mathrm{Hu}$ YH et al., [16], reported a clinical study where 11 adult patients underwent the treatment of TMJ ankylosis with alloplastic prosthesis reconstruction. The mean age was 45 years ( 27 to 62 years). The mean duration of ankylosis was 27 years (3-50 years). Three cases were caused by infection and eight were caused by trauma. There were 7 unilateral cases and 4 bilateral cases. Eight patients had undergone several previous surgeries, including autogenous bone grafts or gap arthroplasty. Four patients with bilateral TMJ ankylosis presented severe mandibular deficiency, with their mandibular branch elongated by the prosthesis. Among them, 2 underwent simultaneous LeFort I osteotomies. The mean follow-up period was 12 to 31 months. There were no reports of infection, fracture or loosening of the prostheses. The mean mouth opening was significantly improved from $5.5 \mathrm{~mm}$ preoperatively to $31.6 \mathrm{~mm}$ postoperatively. Computed tomography during followup at least 6 months after surgery indicated that there was no bone resorption around the screws and no formation of ectopic bone around the artificial condylar heads. In the 4 patients with mandibular branch elongation, there was no significant change in the SNA angle after the operation, but the SNB angle improved significantly from $61.99{ }^{\circ}$ before the operation to $67.86^{\circ}$ after the operation. The heights of the mandibular branches were significantly elongated at $7.25 \mathrm{~mm}$ on the right side and $8.50 \mathrm{~mm}$ on the left postoperative side and the mean advancement of the chin was $10 \mathrm{~mm}$.

According to Khadka and $\mathrm{Hu}$ [40], several autogenous grafts are available for condylar reconstruction after release of the ankylotic mass, such as costochondral, sternoclavicular, fibular, coronary and metatarsal. The costochondral graft is preferred by surgeons, but if compared to osteogenic distraction, it has as main disadvantages donor site morbidity, lack of reabsorption control and remodeling, in this way DO is slowly gaining popularity and may eventually become the standard procedure in the very near future, providing an economical approach with low morbidity and excellent functional results [6, 7, 9-11]. Tissue engineering is another field of field studies that has shown promising results in animal studies but has not yet been applied in humans. To date, there is no ideal autogenous graft for condylar reconstruction due to the 
complex anatomy and myriad functions of a lost condyle.

Compared with autogenous bone grafts, the prosthesis has advantages such as stability and exclusion of morbidity at the donor site, which reduces surgical trauma and shortens operative time. Another advantage of alloplastic prostheses is the low rates of ankylosis recurrence compared to autogenous grafts [14-17].

Because it is a recent technique we can not affirm the success of the prostheses in the long term, and its high cost also acts as a disadvantage to the expansion of its use.

An alternative DO approach has revolutionized the field of craniomaxillofacial reconstructive surgery. This is a versatile technique aimed at modulating new bone growth and involves genetic stimulation in the construction of local tissues to increase in total thickness rather than transfer from elsewhere. In OD, the tissues are submitted to slow and constant physiological traction and become metabolically activated with increased biosynthetic cell function and proliferation. It occurs the generation of new bone in a space created between two bone segments, in response to the application of a tensile stress graded in that space. A unique feature of distraction is bone regeneration where osteogenesis is accompanied by simultaneous expansion of all involved tissues, including bone tissue, skin, muscles, vessels, ligaments, cartilage, and periosteum.

Mandibular distraction has revolutionized the treatment of children with ankylosis and associated micrognathia. These patients present with severe obstruction of the airways. The distraction technique remains the only intervention that directly corrects mandibular hypoplasia and the back tongue, providing efficient relief of airway stenosis. Multiple studies have demonstrated the efficacy of distraction to avoid tracheostomy and decrease the severity of airway obstruction in this group of patients. It is therefore not surprising that distraction mandibular has become the first-line intervention in many centers for the surgical treatment of patients with micrognathia. The reported complications associated with mandibular distraction are relatively low, with infection being the most common and easily treated with antibiotics.

The surgical technique advocated by Flores RL [23, 29, 30, 31, 36], consisted of an extraoral approach through Risdon access for direct exposure of the mandible without risk of contamination with the intraoral environment. After the mandibular exposure, coronoidectomy and vertical osteotomy of the branch were performed and distractors were installed, this type of osteotomy prevents damage to the inferior alveolar bundle and developing dental shoots. Osteotomies may still be inverted $\mathrm{L}$ or mandibular body, depending on the region of the deficiency. In cases where mandibular atresia is in the body and the morphology of the mandible is normal, a horizontal distractor is used, whereas in cases with branch deficiency mandible, the distractor is installed vertically. However, a vertical distractor runs the risk of iatrogenic damage to the temporomandibular joint, due to the positioning of the device and the screws very close to the condyle or indirectly, by the distraction force in the direction of the glenoid fossa.

The ankylosis of TMJ in the child is a morbid complication and a challenging clinical problem, although its incidence has been reported as high in $10 \%$ of cases [23], the author reports that he has managed to avoid this complication through the implementation of a horizontal distractor. Corticotomies were performed in the mandibular body and the horizontal distractors were installed. After a 5-day latency period, activation begins at a rate of $1 \mathrm{~mm}$ per day until the maximum distractor length of $20 \mathrm{~mm}$ is reached. Serial cephalograms are used to monitor progress and confirm bilateral symmetrical advancement. The devices were removed after 8 weeks of consolidation. At the moment of removal of the device, the integrity of the neoformed bone was evaluated by direct visualization and palpation. A satisfactory result was given in $92-100 \%$ of cases of syndromic micrognathia in neonates with severe obstruction of the upper airways treated with osteogenic distraction.

One of the major challenges in the treatment of ATM ankylosis with micrognathia in children is mainly because it is a growing individual, so the surgical techniques employed, as well as their correct sequence, are still very controversial among the authors, each one having reasons to defend their studies [14, 18-21]. Rao $\mathrm{K}$ et al., [18], argues that associating the procedures of joint arthroplasty with the concomitant gap with the OD installation decreases the treatment time and excludes the need for a second surgical intervention, also decreasing the morbidity of the procedures. Other authors, such as Sharma A et al., [12], prefer to first perform ankylosed ATM arthroplasty to later install the distractor in micrognath correction. Sadakah AA et al., [5], presented a modification of this technique where he first installed the distractors in the mandibular branch and associated with a Le Fort 1 osteotomy, with the justification that with a static joint the control of distractor movements and occlusal stability would be more predictable.

With the advancement of miniaturized intraoral distractors, the distraction osteogenesis technique has drastically transformed the concept of correction of maxillofacial deformities [3-7], having multiple advantages over extraoral distractors, considering the patient's better acceptance and compliance during the activation and consolidation 
phases because they are discrete and easy to handle, the reduction of the scar of the skin caused by the traction of transcutaneous fixation pins, preventing injury to the neurovascular bundles, allowing the invisible distraction of the jaw, thus eliminating frequent monitoring and limitation in social life and improving the stability of the attachment to the bone [24-27].

Vertical or horizontal distraction of the mandible branch or body respectively, or the combination of both, may be accomplished through a corticotomy at the desired location and positioning of the intraoral distraction apparatus linearly, vertically or angularly $^{28,29,30}$. After a mean period of 5-7 days postoperative latency to allow soft tissue healing and smooth bone callus formation, the stents on either side of the osteotomy are slowly distracted at a distance of approximately $1 \mathrm{~mm}$ per day, divided into 2 or 3 daily activations until it reaches the desired length and contour. A period of stabilization of the distraction between 8 and 16 weeks is waited until the devices are removed. This modality of treatment is considered relatively conservative in relation to osteotomies with bone grafts for maxillo-mandibular reconstruction, thus avoiding a prolonged hospital stay and allowing early return to routine activities of the patients. It also eliminates the risk and morbidity associated with the donor site of autogenous grafts, need for implants and problems of incompatibility, as well as the absence of need for maxillo-mandibular fixation.

As native bone is created, it allows the surgeon to have postoperative control so that the distraction process is stopped at any point according to the requirement of each case 4 . It also provides good term stability, since locally regenerated original bone is created along with proper neuromuscular readaptation, thus yielding cosmetic results that are far superior to those obtained by either skeletal or soft tissue surgery done independently, or by the combination of both $31,32,33,34,35$.

Regarding the techniques of ankylosis surgery we have a variety of procedures both for resection of the ankylosis mass and reconstruction of the joint. Giraddi BG et al., [17, 35, 36, 38], 2017, preferred in his clinical study to perform the gap arthroplasty interposing in all cases a temporal myofascial flap flap and did not obtain any case of relapse in its results. Bansal et al., [37-39], in their cases performed the gap arthroplasty without interposing any material and used OD for reconstruction of the resected condyle, which after an average of 19 postoperative months, reached the result of remodeling of the condyles and restoration of the joints also with no history of relapse.

In the cases reported, the strategy was adopted to remove the ankylotic block without interposition of muscle or other material, and the distractors were installed at the same surgical time. We believe that in this way we will contribute to the development of soft tissues, in addition to bone tissue, reducing possible sequelae in relation to facial development. The major difficulties faced were patient and family members adherence to intensive physical therapy, and skin infections due to the presence of distractors. There was no report of pain during device activation.
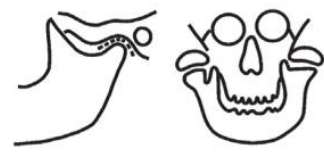

Type I

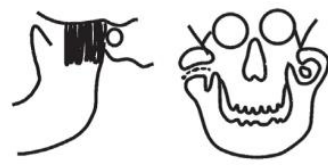

Type III

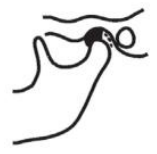

Typell
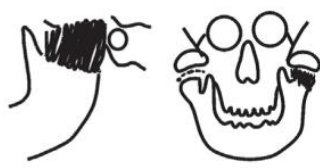

Type IV
Fig-1: Ranking Sawnhey by Turlington and Durr [2]
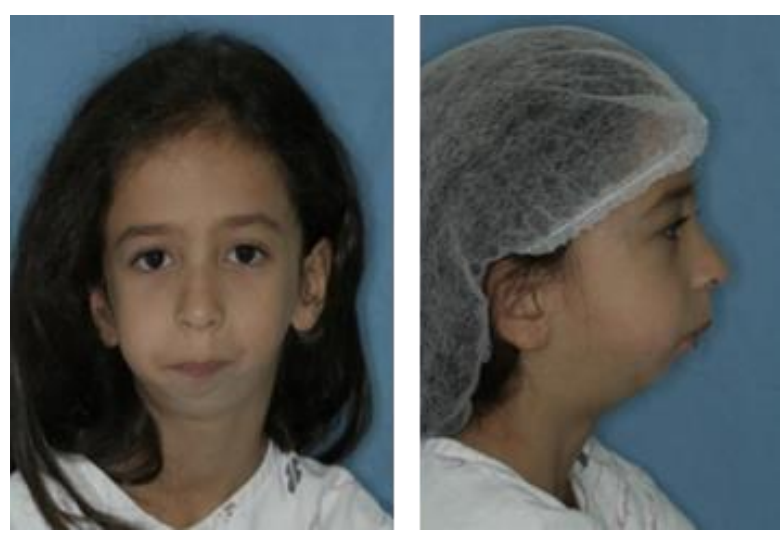

Fig-2 : Front and perfil view (Carlini 2015)
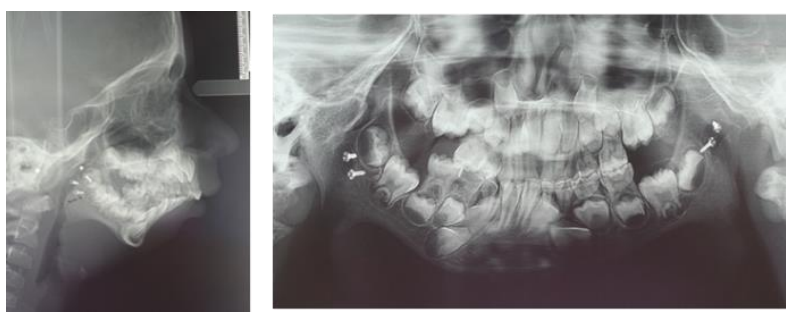

Fig-3: Initial Teleradiography and Panormamica (Carlini 2015) 
João Luiz Carlini et al; Saudi J Oral Dent Res, Oct 2019; 4(10): 726-737

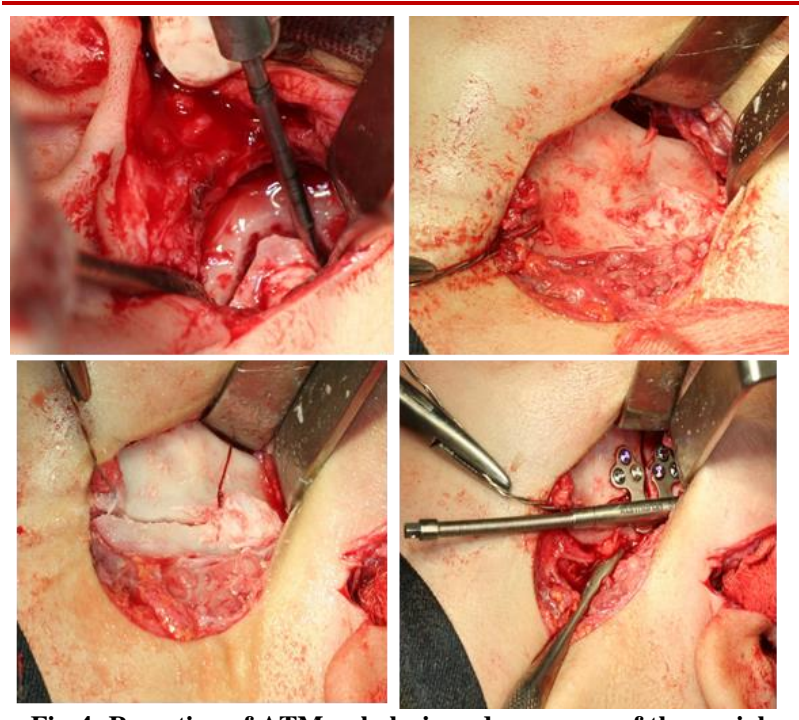

Fig-4: Resection of ATM ankylosis and exposure of the gonial angle, Osteotomy of inverted L-mandibular and Installation of the linear osteogenic distractor (Carlini 2015)
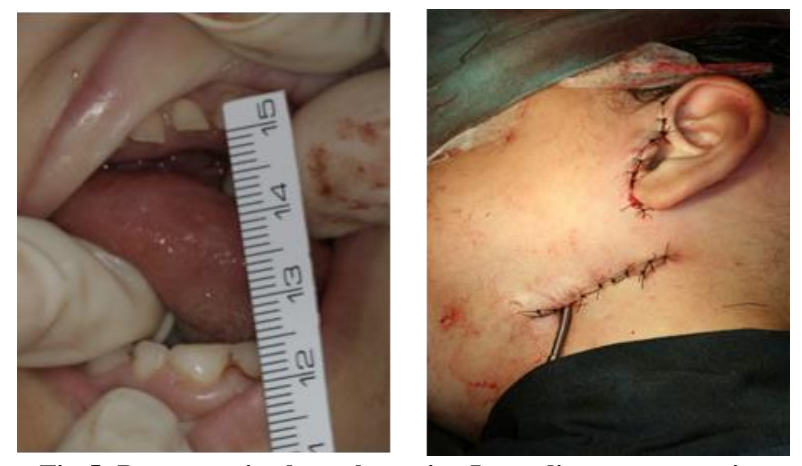

Fig-5: Postoperative buccal opening Immediate postoperative (Carlini 2015)

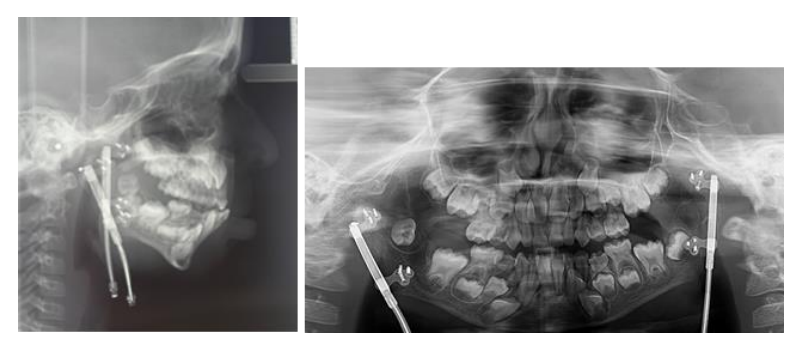

Fig-6: Teleradiography profile after 90 days and Panoramic $\mathrm{X}$ ray (Carlini 2016)
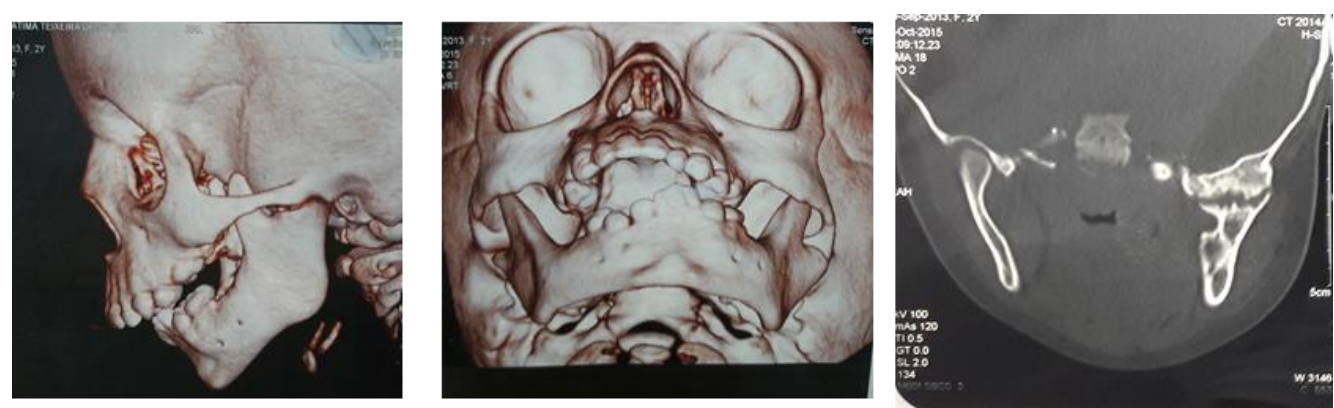

Fig-9: Initial tomography (CAIF 2015)

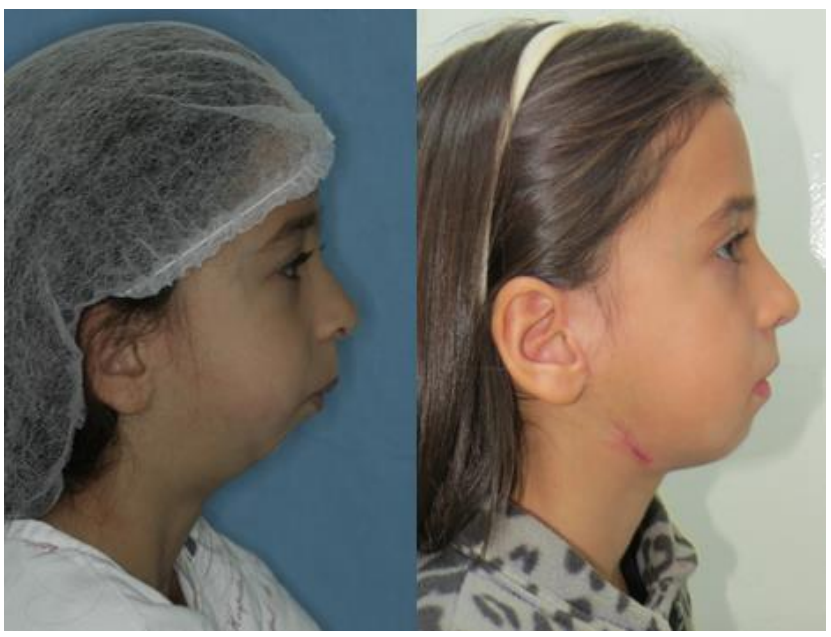

Fig-7: Preoperative aspect and final appearance (Carlini 2017)

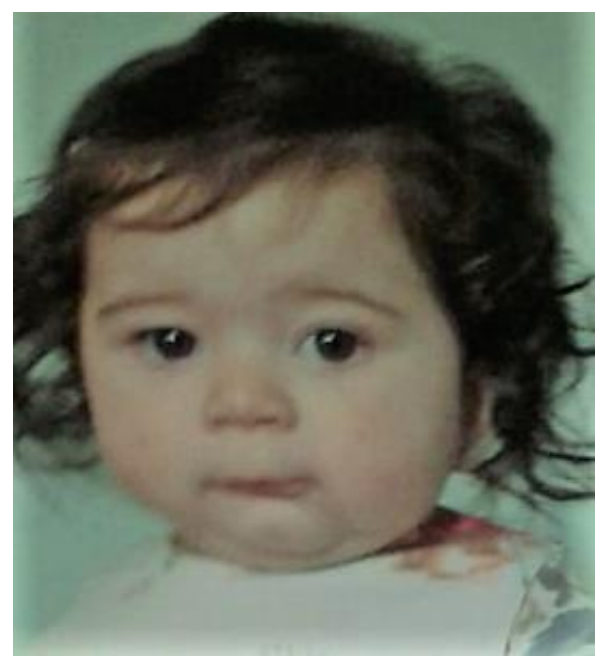

Fig-8: Initial appearance (family file 2015) 

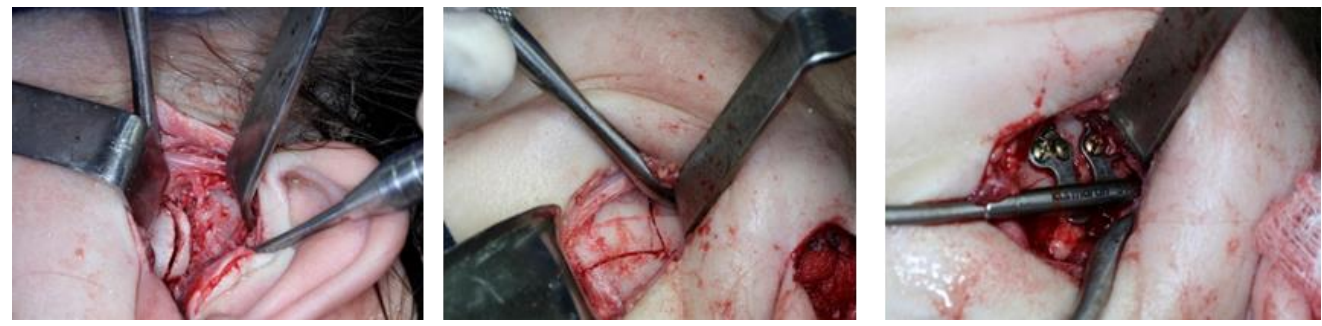

Fig-10: Removal of ankylosis mass, inverted L- osteotomy and distractor installation (Carlini 2016)
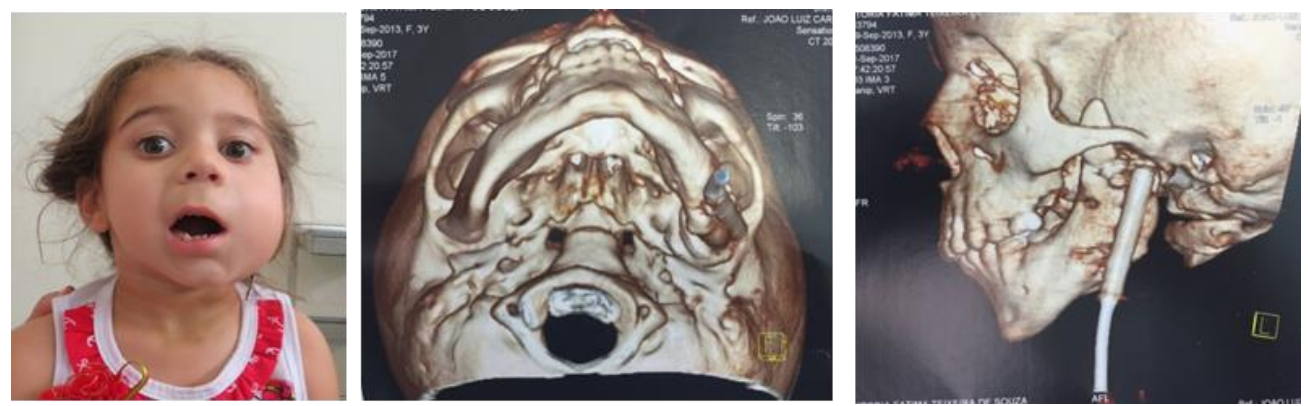

Fig-11: Postoperative after total distraction of 1st surgery (Carlini 2016)
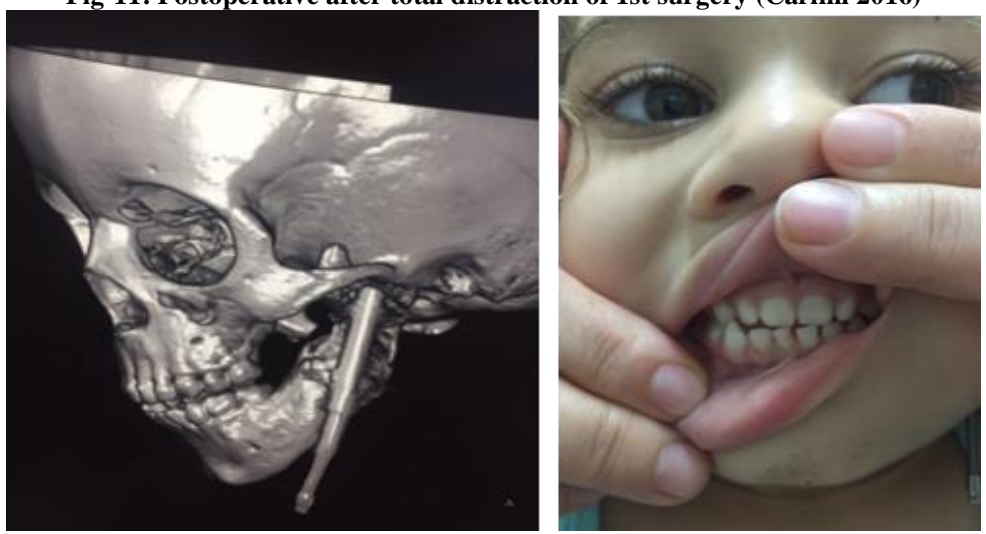

Fig-12: Tomographic after reinstalled the distractor, correct midline (Carlini 2017)
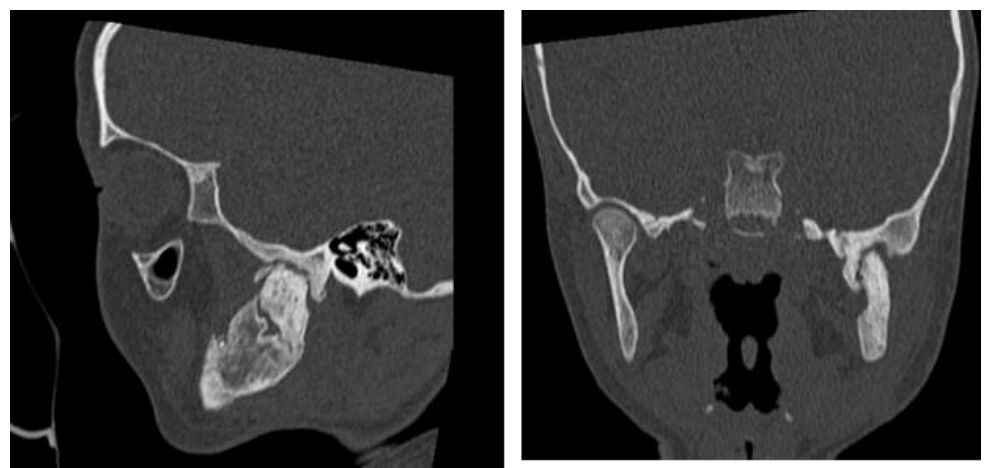

Fig-13: Vista sagital Vista coronal (CAIF 2018)

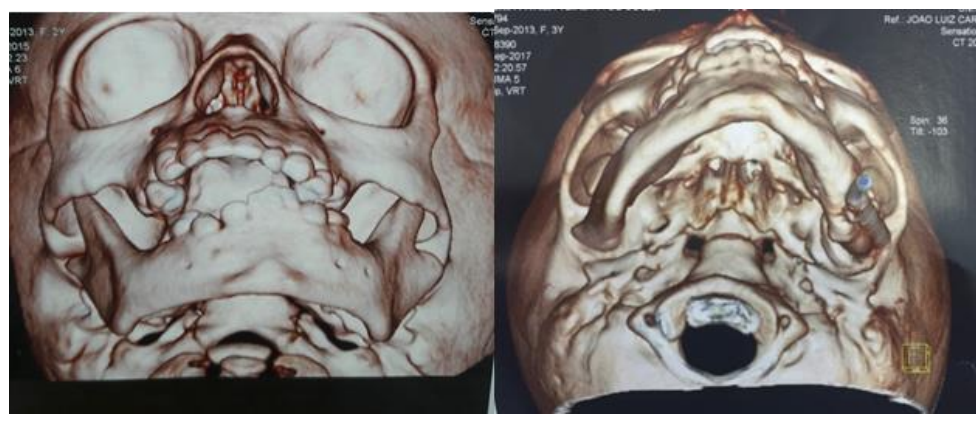

Fig-14: Pre and post operative with midline correction (Carlini 2017 

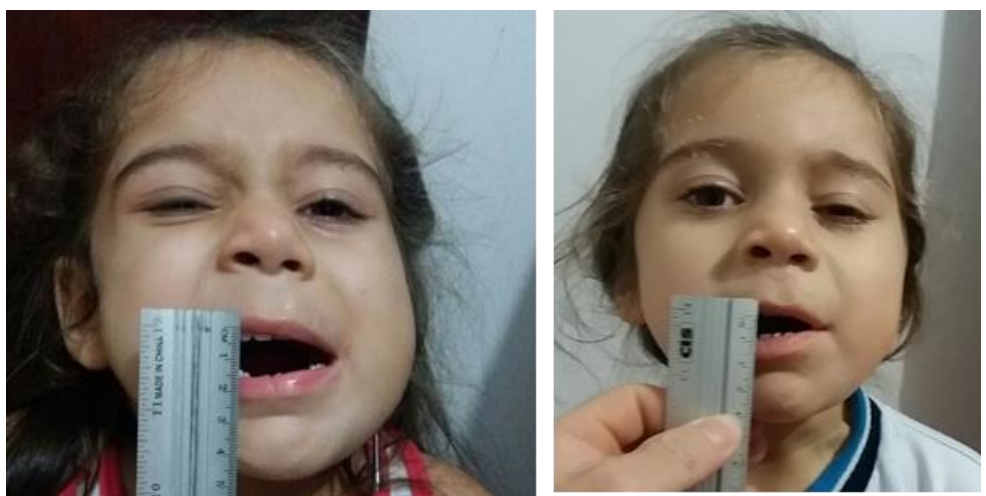

Fig-15: Mouth opening after 3rd surgery of $15 \mathrm{~mm}$ and after removed distractor (10mm) (Carlini 2018)

\section{CONCLUSION}

Although there is no consensus about an ATM ankylosis treatment protocol in children, we observed in this study:

The sooner intervene the results for the soft tissues facilitating facial development and minimizing asymmetry;

Osteogenic distraction is a good treatment option for ankylosis in children, as it induces uniform bone growth and development, as well as soft tissue suitable for bone formation, improving speech, swallowing and chewing, as well as social life;

Family adherence is very important in physiotherapy to maintain the outcome.

\section{REFERENCES}

1. Harshbarger III, R. J. (2014, November). Craniomaxillofacial Distraction Osteogenesis. In Seminars in plastic surgery, 28(4), 161-162. Thieme Medical Publishers.

2. Sporniak-Tutak, K., Janiszewska-Olszowska, J., \& Kowalczyk, R. (2011). Management of temporomandibular ankylosis-compromise or individualization-a literature review. Medical science monitor: international medical journal of experimental and clinical research, 17(5), RA111116.

3. Trahar, M., Sheffield, R., Kawamoto, H., Lee, H. F., \& Ting, K. (2003). Cephalometric evaluation of the craniofacial complex in patients treated with an intraoral distraction osteogenesis device: a preliminary report. American journal of orthodontics and dentofacial orthopedics, 124(6), 639-650.

4. Sapru, B. L. (2001). Distraction Osteogenesis" An Emerging Concept In The Correction Of Craniofacial Deformities. Medical journal, Armed Forces India, 57(4), 273.

5. Sadakah, A. A., Elgazzar, R. F., \& Abdelhady, A. I. (2006). Intraoral distraction osteogenesis for the correction of facial deformities following temporomandibular joint ankylosis: a modified technique. International journal of oral and maxillofacial surgery, 35(5), 399-406.

6. Balaji, S. M. (2009). Teeth position, eruption and temporomandibular joint ankylosis. Journal of maxillofacial and oral surgery, 8(4), 340-343.

7. Heggie, A. A., Kumar, R., \& Shand, J. M. (2013). The role of distraction osteogenesis in the management of craniofacial syndromes. Annals of maxillofacial surgery, 3(1), 4 .

8. Feiyun, P., Wei, L., Jun, C., Xin, X., Zhuojin, S., \& Fengguo, Y. (2010). Simultaneous correction of bilateral temporomandibular joint ankylosis with mandibular micrognathia using internal distraction osteogenesis and 3-dimensional craniomaxillofacial models. Journal of Oral and Maxillofacial Surgery, 68(3), 571-577.

9. Aneja, V., Raval, R., Bansal, A., Kumawat, V., Kaur, J., \& Shaikh, A. A. (2016). Interpositional Gap Arthroplasty by Versatile Pedicled Temporalis Myofascial Flap in the Management of Temporomandibular Joint Ankylosis-A Case Series Study. Journal of clinical and diagnostic research: JCDR, 10(10), ZR01- ZR04.

10. Donneys, A., Deshpande, S. S., Tchanque-Fossuo, C. N., Johnson, K. L., Blough, J. T., Perosky, J. E., ... \& Levi, B. (2013). Deferoxamine expedites consolidation during mandibular distraction osteogenesis. Bone, 55(2), 384-390.

11. Newbury, P. A., Adams, N. S., \& Girotto, J. A. (2015). Mandibular Distraction Osteogenesis: Upper Airway Management in Pierre Robin Sequence. Eplasty, 15.

12. Sharma, A., Paeng, J. Y., Yamada, T., \& Kwon, T. G. (2016). Simultaneous gap arthroplasty and intraoral distraction and secondary contouring surgery for unilateral temporomandibular joint ankylosis. Maxillofacial plastic and reconstructive surgery, 38(1), 12.

13. Gaur, A., Singh, G., Mishra, M., Srivatsan, K. S., \& Sachdev, V. (2013). Distraction osteogenesis for management of obstructive sleep apnea secondary to TMJ ankylosis. National journal of maxillofacial surgery, 4(1), 104. 
14. Anantanarayanan, P., \& Manikandhan, R. (2013). Cephalometric evaluation of airway changes following mandibular distraction in patients with nocturnal desaturations during sleep secondary to TMJ ankylosis. Journal of maxillofacial and oral surgery, 12(1), 17-20.

15. Gupta, N., Gupta, N., Tomar, L. R., \& Nair, N. (2016). Temporomandibular joint ankylosis in ankylosing spondylitis: A case report and review of literature. Journal of family medicine and primary care, 5(3), 716-718.

16. Hu, Y., Zhang, L., He, D., Yang, C., Chen, M., Zhang, S., ... \& Ellis III, E. (2017). Simultaneous treatment of temporomandibular joint ankylosis with severe mandibular deficiency by standard TMJ prosthesis. Scientific reports, 7, 45271.

17. Giraddi, G. B., Arora, K., \& Anusha, A. S. (2016). Distraction osteogenesis in the treatment of temporomandibular joint ankylosis with mandibular micrognathia. Annals of maxillofacial surgery, 6(1), 68.

18. Rao, K., Kumar, S., Kumar, V., Singh, A. K., \& Bhatnagar, S. K. (2004). The role of simultaneous gap arthroplasty and distraction osteogenesis in the management of temporo-mandibular joint ankylosis with mandibular deformity in children. Journal of Cranio-Maxillofacial Surgery, 32(1), 38-42.

19. Kaban, L. B., Seldin, E. B., Kikinis, R., Yeshwant, K., Padwa, B. L., \& Troulis, M. J. (2009). Clinical application of curvilinear distraction osteogenesis for correction of mandibular deformities. Journal of Oral and Maxillofacial Surgery, 67(5), 9961008.

20. Cheong, R. C. T., Kassam, K., Eccles, S., \& Hensher, R. (2016). Congenital temporomandibular joint ankylosis: case report and literature review. Case reports in otolaryngology, 2016.

21. Ma, J., Liang, L., Jiang, H., \& Gu, B. (2015). Gap arthroplasty versus interpositional arthroplasty for temporomandibular joint ankylosis: a metaanalysis. PloS one, 10(5), e0127652.

22. Cho, J. W., Park, J. H., Kim, J. W., \& Kim, S. J. (2016). The sequential management of recurrent temporomandibular joint ankylosis in a growing child: a case report. Maxillofacial plastic and reconstructive surgery, $38(1), 39$.

23. Flores, R. L. (2014, November). Neonatal mandibular distraction osteogenesis. In Seminars in plastic surgery, 28(4) 199-206. Thieme Medical Publishers.

24. Karun, V., Agarwal, N., \& Singh, V. (2013). Distraction osteogenesis for correction of mandibular abnormalities. National journal of maxillofacial surgery, 4(2), 206.

25. Yew, C. C., Ab Rahman, S., \& Alam, M. K. (2015). Temporomandibular joint ankylosis in a child: an unusual case with delayed surgical intervention. BMC pediatrics, 15(1), 169.
26. Sharma, H., Chowdhury, S., Navaneetham, A., Upadhyay, S., \& Alam, S. (2015). Costochondral graft as interpositional material for TMJ ankylosis in children: a clinical study. Journal of maxillofacial and oral surgery, 14(3), 565-572.

27. Sesenna, E., Magri, A. S., Magnani, C., Brevi, B. C., \& Anghinoni, M. L. (2012). Mandibular distraction in neonates: indications, technique, results. Italian Journal of Pediatrics, 38(1), 7.

28. Tomonari, H., Takada, H., Hamada, T., Kwon, S., Sugiura, T., \& Miyawaki, S. (2017). Micrognathia with temporomandibular joint ankylosis and obstructive sleep apnea treated with mandibular distraction osteogenesis using skeletal anchorage: a case report. Head \& face medicine, 13(1), 20.

29. Parmar, B. S., Garg, B., Mehta, R. D., Midha, A., \& Thakkar, D. K. (2015). Ramus condyle unit reconstruction using vertical ramus osteotomy in temporomandibular joint ankylosis. Journal of maxillofacial and oral surgery, 14(3), 630-636.

30. Swennen, G., Schliephake, H., Dempf, R., Schierle, H., \& Malevez, C. (2001). Craniofacial distraction osteogenesis: a review of the literature. Part 1: clinical studies. International journal of oral and maxillofacial surgery, 30(2), 89-103.

31. Bansal, V., Singh, S., Garg, N., \& Dubey, P. (2014). Transport distraction osteogenesis as a method of reconstruction of the temporomandibular joint following gap arthroplasty for post-traumatic ankylosis in children: a clinical and radiological prospective assessment of outcome. International journal of oral and maxillofacial surgery, 43(2), 227-236.

32. Silva, L. M. D. C., Pereira Filho, V. A., Vieira, E. H., \& Gabrielli, M. F. R. (2011). Tracheostomydependent child with temporomandibular ankylosis and severe micrognathia treated by piezosurgery and distraction osteogenesis: case report. British Journal of Oral and Maxillofacial Surgery, 49(7), e47-e49.

33. López, E. N., \& Dogliotti, P. L. (2004). Treatment of temporomandibular joint ankylosis in children: is it necessary to perform mandibular distraction simultaneously?. Journal of Craniofacial Surgery, 15(5), 879-884.

34. Sahoo, N. K., Tomar, K., Kumar, A., \& Roy, I. D. (2012). Selecting reconstruction option for TMJ ankylosis: a surgeon's dilemma. Journal of Craniofacial Surgery, 23(6), 1796-1801.

35. Elgazzar, R. F., Abdelhady, A. I., Saad, K. A., Elshaal, M. A., Hussain, M. M., Abdelal, S. E., \& Sadakah, A. A. (2010). Treatment modalities of TMJ ankylosis: experience in Delta Nile, Egypt. International journal of oral and maxillofacial surgery, 39(4), 333-342.

36. Bartlett, S. P., Losee, J. E., \& Quinn, P. D. (2006). Severe proliferative congenital temporomandibular joint ankylosis: a proposed treatment protocol utilizing distraction osteogenesis. Journal of Craniofacial Surgery, 17(3), 605-610. 
37. Zhang, C., Li, Y., Ye, B., Liu, Y., Bao, C., \& Zhu, S. (2017). Application of bidirectional distraction osteogenesis for the treatment of mandibular micriognathia caused by temporomandibular joint ankylosis. Journal of Craniofacial Surgery, 28(6), 1502-1507.

38. Cascone, P., Agrillo, A., Spuntarelli, G., Arangio, P., \& Iannetti, G. (2002). Combined surgical therapy of temporomandibular joint ankylosis and secondary deformity using intraoral distraction. Journal of Craniofacial Surgery, 13(3), 401-409.
39. Papageorge, M. B., \& Apostolidis, C. (1999). Simultaneous mandibular distraction and arthroplasty in a patient with temporomandibular joint ankylosis and mandibular hypoplasia. Journal of oral and maxillofacial surgery, 57(3), 328-333.

40. Khadka, A., \& Hu, J. (2012). Autogenous grafts for condylar reconstruction in treatment of TMJ ankylosis: current concepts and considerations for the future. International journal of oral and maxillofacial surgery, 41(1), 94-102. 